\title{
ORGANIZAÇÕES E PESSOAS PARTICIPANTES DO CICLO DE PREMIAÇÃO 2002
}

Encerrado o período de inscrições para o Ciclo de Premiação 2002 do Programa GESTÃo PÚBLICA E CIDADANIA, 981 programas, projetos ou atividades haviam enviado material e estavam aptos a participar do processo seletivo para a fase subseqüente.

A partir daí, a análise do material mobilizou uma Comissão de Seleção formada por membros de organizações da sociedade civil e pesquisadores de universidades e de instituições de pesquisa, todos eles de alguma forma atuantes na busca da melhoria da administração pública brasileira. Subdividido em grupos de quatro, esta Comissão organizou-se de forma a garantir que cada iniciativa governamental inscrita fosse analisada e discutida, preliminarmente, por dois grupos diferentes. A partir daí, uma nova rodada de discussão geral, na qual cada caso foi exaustivamente debatido, permitiu que se chegasse à indicação dos semifinalistas com base na construção gradual de consensos.

Uma vez indicados os 100 semifinalistas, foi-lhes solicitado responder a um questionáriopadrão, composto de 18 perguntas (ver quadro III, p.20). Estas respostas subsidiaram a análise para indicação dos 30 pré-finalistas, em processo idêntico ao da etapa anterior. Escolhidas estas 30 iniciativas, um conjunto de especialistas e estudiosos foi a campo conhecer as experiências in loco, preparando então os "Relatórios de Visita de Campo". Estes relatórios possibilitaram a escolha dos 20 finalistas, em uma nova bateria de discussões. Todo o material recolhido e produzido ao longo deste processo passou a compor o Banco de Dados do Programa, estando disponivel a qualquer interessado para consulta e obtenção de cópias.

O Ciclo de Premiação 2002 será encerrado com um evento realizado na sede do Banco Nacional de Desenvolvimento Econômico e Social - BNDES, no Rio de Janeiro, no qual os responsáveis pelas 20 iniciativas finalistas apresentarão ao público presente e debaterão com uma Banca Julgadora os programas desenvolvidos pelas organizações públicas das quais fazem parte. Esta Banca se reunirá ao final do dia e indicará cinco destaques.

A seguir, apresentamos as organizações e pessoas que de forma mais ativa estiveram envolvidas em cada uma das etapas deste esforço coletivo para identificar e disseminar os caminhos que vêm sendo percorridos rumo a uma melhor administração pública. 


\section{COORDENAÇÃO DO PROGRAMA}

\begin{tabular}{|c|c|}
\hline Elizabeth Leeds & $\begin{array}{l}\text { - Assessora do Programa de Governo e Sociedade Civil } \\
\text { Fundação Ford no Brasil }\end{array}$ \\
\hline Peter Spink & $\begin{array}{l}\text { - Diretor do Programa GESTÃO PÚBLICA E CIDADANIA } \\
\text { Curso de Mestrado em Administração Pública e Governo } \\
\text { Centro de Estudos de Administração Pública e Governo } \\
\text { Escola de Administração de Empresas de São Paulo } \\
\text { Fundação Getulio Vargas - EAESP/FGV }\end{array}$ \\
\hline Marta Ferreira Santos Farah & $\begin{array}{l}\text { Vice-Diretora do Programa GESTÃo PÚBLICA E } \\
\text { CIDADANIA } \\
\text { Centro de Estudos de Administração Pública e Governo } \\
\text { Escola de Administração de Empresas de São Paulo } \\
\text { Fundação Getulio Vargas - EAESP/FGV }\end{array}$ \\
\hline Fernando Gilherme Tenório & - Profesor da Fundação Getulio Vargas - EBAP/FGV \\
\hline Ricardo Bresler & - Vice- Coordenador do curso de graduação - EAESP/FGV \\
\hline llka Camarotti & $\begin{array}{l}\text { - Coordenadora Técnica do Subprograma Práticas Públicas e } \\
\text { Pobreza }\end{array}$ \\
\hline Fabiana P. Sanches de Moura & $\begin{array}{l}\text { - Coordenadora Administrativa do Programa Gestão Pública e } \\
\text { Cidadania }\end{array}$ \\
\hline Marlei de Oliveira & - Secretária do Programa Gestão Pública e Cidadania \\
\hline Juliana Maria Paris Spink & - Assistente de Vídeo e Disseminação \\
\hline Marco Antonio C. Teixeira & Pesquisador \\
\hline Jacqueline I. M. Brigagão & Pesquisadora \\
\hline Rafael Marques Osório & - Monitor Acadêmico \\
\hline Fernanda Oliveira & - Monitora Acadêmica \\
\hline Hélio Batista Barboza & - Monitor Acadêmico \\
\hline Luis Mario Fujiwara & - Monitor Acadêmico \\
\hline Francine Lemos Arouca & - Monitora Acadêmica \\
\hline
\end{tabular}




\section{COMITÉ TÉCNICO}

\begin{tabular}{|c|c|}
\hline Edna M. Ramos de Castro & $\begin{array}{l}\text { - Núcleos de Altos Estudos Amazônicos - Universidade } \\
\text { Federal do Pará }\end{array}$ \\
\hline Elizabeth Leeds & - Fundação Ford \\
\hline Franklin Coelho & - Universidade Federal Fulminense / Viva Rio \\
\hline Humberto Marques Filho & $\begin{array}{l}\text { - Programa de Pós-Graduação em Administração - } \\
\text { Universidade Federal da Paraíba }\end{array}$ \\
\hline $\begin{array}{l}\text { José Antônio Gomes de } \\
\text { Pinho }\end{array}$ & $\begin{array}{l}\text { - Núcleo de Pesquisa e Pós-Graduação em Administração - } \\
\text { Universidade Federal da Bahia }\end{array}$ \\
\hline Laura da Veiga & $\begin{array}{l}\text { - Escola de Governo de Minas Gerais - Fundação João } \\
\text { Pinheiro }\end{array}$ \\
\hline Luis Roque Klering & $\begin{array}{l}\text { - Programa de Pós-Graduação em Administração - } \\
\text { Universidade Federal do Rio Grande do Sul }\end{array}$ \\
\hline Marlene Libardoni & $\begin{array}{l}\text { - Ações em Gênero, Cidadania e Desenvolvimento - } \\
\text { AGENDE }\end{array}$ \\
\hline Marta Prochnik & - Banco de Desenvolvimento Econômico e Social - BNDES \\
\hline Nancy Cardia & - Núcleo de Estudos da Violência - USP \\
\hline Paulo Pankararu & $\begin{array}{l}\text { - Coordenação das Organizações Indigenas da Amazônia } \\
\text { Brasileira (COIAB) }\end{array}$ \\
\hline Pedro Jacobi & $\begin{array}{l}\text { - Programa de Pós-Graduação em Ciência Ambiental da } \\
\text { Universidade de São Paulo (Procam/USP) }\end{array}$ \\
\hline Silvio Caccia Bava & $\begin{array}{l}\text { - Pólis - Instituto de Estudos, Formação e Assistência em } \\
\text { Políticas Sociais }\end{array}$ \\
\hline Sueli Carneiro & - GELEDES - Instituto da Mulher Negra \\
\hline
\end{tabular}




\section{COMISSÃO DE SELEÇÃO - $1^{a}$ FASE (INDICAÇÃO DOS SEMIFINALISTAS)}

\begin{tabular}{|c|c|}
\hline Ana Maria Malik & - Professora EAESP/FGV \\
\hline Ana Paula Karruz & - Mestranda em Administração Pública e Governo - EAESP/FGV \\
\hline Antonio Faria da Costa & - Mestrando em Administração Pública e Governo - EAESP/FGV \\
\hline Antônio Sérgio Fernandes & - Doutorando em Ciência Política - USP \\
\hline Cristina Andrews & - Doutora em Ciência Política - FHLCH/USP \\
\hline Edna M. Ramos de Castro & - Núcleo de Altos Estudos Amazônicos - Universidade Federal do Pará \\
\hline Eduardo de Lima Caldas & - Mestrando em Administração Pública e Governo - EAESP/FGV \\
\hline Fátima Timóteo & - Mestranda em Administração Pública e Governo - EAESP/FGV \\
\hline Fernando Araújo & - Mestrando em Administração Pública e Governo - EAESP/FGV \\
\hline Flávio Calife & - Doutorando em Administração Pública e Governo - EAESP/FGV \\
\hline Franklin Coelho & - Universidade Federal Fulminense / Viva Rio \\
\hline $\begin{array}{l}\text { Fernando Guilherme } \\
\text { Tenório }\end{array}$ & $\begin{array}{l}\text { - Escola Brasileira de Administração Pública - Fundação Getulio Vargas - } \\
\text { EBAP/FGV }\end{array}$ \\
\hline Guilherme Paula e Silva & - Mestrando em Administração Pública e Governo - EAESP/FGV \\
\hline Heitor Paulo Battaglia & - Mestre em Administração Pública e Governo - EAESP/FGV \\
\hline Humberto Marques Filho & $\begin{array}{l}\text { - Programa de Pós-Graduação em Administração - } \\
\text { Universidade Federal da Paraiba }\end{array}$ \\
\hline Ilka Camarotti & - Subprograma Práticas Públicas e Pobreza - EAESP/FGV \\
\hline Iraci Ózeas dos Reis & $\begin{array}{l}\text { - Doutoranda em Serviço Social na Pontificia Universidade Católica de } \\
\text { São Paulo - PUC-SP }\end{array}$ \\
\hline Janaína V. de Mattos & $\begin{array}{l}\text { - Pólis - Instituto de Estudos, Formação e Assessoria em Políticas } \\
\text { Sociais }\end{array}$ \\
\hline Jorge E. Ruiz & - Mestrando em Administração Pública e Governo - EAESP/FGV \\
\hline $\begin{array}{l}\text { José Antônio Gomes de } \\
\text { Pinho }\end{array}$ & $\begin{array}{l}\text { - Núcleo de Pesquisa e Pós-Graduação em Administração - } \\
\text { Universidade Federal da Bahia }\end{array}$ \\
\hline José Carlos Vaz & $\begin{array}{l}\text { - Pólis - Instituto de Estudos, Formação e Assessoria em Políticas } \\
\text { Sociais }\end{array}$ \\
\hline Luciane P. D'ávila Melo & - Mestrando em Administração Pública e Governo - EAESP/FGV \\
\hline Luis Mario Fujiwara & - Mestrando em Administração Pública e Governo - EAESP/FGV \\
\hline Luis Roque Klering & - Programa de Pós-Graduação em Administração - UFRS \\
\hline Marco Antônio Teixeira & $\begin{array}{l}\text { - Doutorando em Ciência Política na Pontifícia Universidade Católica de } \\
\text { São Paulo - PUC/SP }\end{array}$ \\
\hline Maria Aparecida da Silva & - GELEDES - Instituto da Mulher Negra \\
\hline Maria do C. Meirelles & - Fundação Prefeito Faria Lima -.CEPAM \\
\hline Marlene Libardoni & - Mestrando em Administração Pública e Governo - EAESP/FGV \\
\hline Nancy Cardia & - Núcleo de Estudos da Violência - USP \\
\hline Natalia Koga & - Mestranda em Administração Pública e Governo - EAESP/FGV \\
\hline Otávio Prado & - Mestrando em Administração Pública e Governo - EAESP/FGV \\
\hline
\end{tabular}




\begin{tabular}{ll}
\hline \hline Pedro Jacobi & - Programa de Pós-Graduação em Ciência Ambiental da Universidade de \\
Ricardo Bresler & São Paulo (Procam/USP) \\
Rosângela l. da Silva & - Banco Nacional de Desenvolvimento Econômico e Social \\
Silvia Craveiro & - Mestranda em Administração Pública e Governo - EAESP/FGV \\
Silvia Kawata & - Mestrando em Administração Pública e Governo - EAESP/FGV \\
Solange Sanches & - DIEESE \\
Vilmar Guarany & - Organizações Indígenas da Amazônia Brasileira (COIAB) \\
\hline
\end{tabular}


COMISSÃO DE SELEÇÃO - 2a FASE (INDICAÇÃO DOS PRÉ-FINALISTAS)

\begin{tabular}{|c|c|}
\hline Ana Marcia Ramos & - Mestre em Administração Pública e Governo - EAESP/FGV \\
\hline Ana Maria Malik & Professora EAESP/FGV \\
\hline Antonio Faria de Costa & Mestre em Administração Pública e Governo - EAESP/FGV \\
\hline Edna M. Ramos de Castro & $\begin{array}{l}\text { Núcleo de Altos Estudos Amazônicos - Universidade Federal } \\
\text { do Pará }\end{array}$ \\
\hline Eduardo Caldas & - Mestre em Administração Pública e Governo - EAESP/FGV \\
\hline Fernanda Oliveira & - Graduanda em Administração Pública - EAESP/FGV \\
\hline Fernando Guilherme Tenório & $\begin{array}{l}\text { - Escola Brasileira de Administração Pública - } \\
\text { Fundação Getulio Vargas - EBAP/FGV }\end{array}$ \\
\hline $\begin{array}{l}\text { Francine Lemos Rodarte } \\
\text { Arouca }\end{array}$ & - Graduanda em Administração Pública - EAESP/FGV \\
\hline Hélio Batista Barboza & $\begin{array}{l}\text { - Mestrando em Administração Pública e Governo - } \\
\text { EAESP/FGV }\end{array}$ \\
\hline Humberto Marques Filho & $\begin{array}{l}\text { - Programa de Pós-Graduação em Administração - } \\
\text { Universidade Federal da Paraíba }\end{array}$ \\
\hline Illka Camarotti & - Subprograma Práticas Públicas e Pobreza - EAESP/FGV \\
\hline Iraci Ózeas dos Reis & $\begin{array}{l}\text { - Doutoranda em Serviço Social na } \\
\text { Pontifícia Universidade Católica de São Paulo - PUC-SP }\end{array}$ \\
\hline $\begin{array}{l}\text { José Antônio Gomes de } \\
\text { Pinho }\end{array}$ & $\begin{array}{l}\text { - Núcleo de Pesquisa e Pós-Graduação em Administração - } \\
\text { Universidade Federal da Bahia }\end{array}$ \\
\hline Laura da Veiga & - Escola de Governo / Fundação João Pinhero \\
\hline Luis Mario Fujiwara & - Mestre em Administração Pública e Governo - EAESP/FGV \\
\hline Luis Roque Klering & $\begin{array}{l}\text { - Programa de Pós-Graduação em Administração - } \\
\text { Universidade Federal do Rio Grande do Sul }\end{array}$ \\
\hline Marco Antônio Teixeira & $\begin{array}{l}\text { - Doutorando em Ciência Política na Pontifícia Universidade } \\
\text { Católica de São Paulo - PUC/SP }\end{array}$ \\
\hline Maria Aparecida & - Geledes - Instituto da Mulher Negra \\
\hline Marlene Libardoni & - Ações em Gênero Cidadania e Desenvolvimento.- AGENDE \\
\hline Paulo Pankararu & $\begin{array}{l}\text { - Coordenação das Organizações Indigenas da Amazônia } \\
\text { Brasilera }\end{array}$ \\
\hline Pedro Jacobi & $\begin{array}{l}\text { - Programa de Pós-Graduação em Ciência Ambiental da } \\
\text { Universidade de São Paulo (Procam/USP) }\end{array}$ \\
\hline osângela I. da Silva & - Banco Nacional de Desenvolvimento Econômico e Social \\
\hline Ivia Regina Costa Salgado & - Fundação Prefeito Faria Lima - CEPAM \\
\hline
\end{tabular}


COMISSÃO DE SELEÇÃO - $3^{\text {a }}$ FASE (INDICAÇÃO DOS FINALISTAS)

\begin{tabular}{|c|c|}
\hline Fernando Guilherme Tenório & $\begin{array}{l}\text { - Escola Brasileira de Administração Pública - } \\
\text { Fundação Getulio Vargas - EBAP/FGV }\end{array}$ \\
\hline Franklin coelho & $\begin{array}{l}\text { - Universidade Federal Fluminense - UFF } \\
\text { Viva Rio }\end{array}$ \\
\hline Humberto Marques Filho & $\begin{array}{l}\text { - Programa de Pós-Graduação em Administração - } \\
\text { Universidade Federal da Paraíba }\end{array}$ \\
\hline llka Camarotti & - Subprograma Práticas Públicas e Pobreza - EAESP/FGV \\
\hline Isys Pagy & - Banco Nacional de Desenvolvimento Econômico e Social \\
\hline $\begin{array}{l}\text { José Antônio Gomes de } \\
\text { Pinho }\end{array}$ & $\begin{array}{l}\text { - Núcleo de Pesquisa e Pós-Graduação em Administração - } \\
\text { Universidade Federal da Bahia }\end{array}$ \\
\hline Laura da Veiga & $\begin{array}{l}\text { - Escola de Governo de Minas Gerais - Fundação João } \\
\text { Pinheiro }\end{array}$ \\
\hline Luis Roque Klering & $\begin{array}{l}\text { - Programa de Pós-Graduação em Administração - } \\
\text { Universidade Federal do Rio Grande do Sul }\end{array}$ \\
\hline Marlene Libardoni & $\begin{array}{l}\text { - AGENDE - Ações em Gênero, Cidadania e } \\
\text { Desenvolvimento }\end{array}$ \\
\hline Pedro Jacobi & $\begin{array}{l}\text { - Programa de Pós-Graduação em Ciência Ambiental da } \\
\text { Universidade de São Paulo (Procam/USP) }\end{array}$ \\
\hline Silvio Caccia Bava & $\begin{array}{l}\text { - Pólis - Instituto de Estudos, Formação e Assessoria em } \\
\text { Políticas Sociais }\end{array}$ \\
\hline
\end{tabular}


VISITAS DE CAMPO

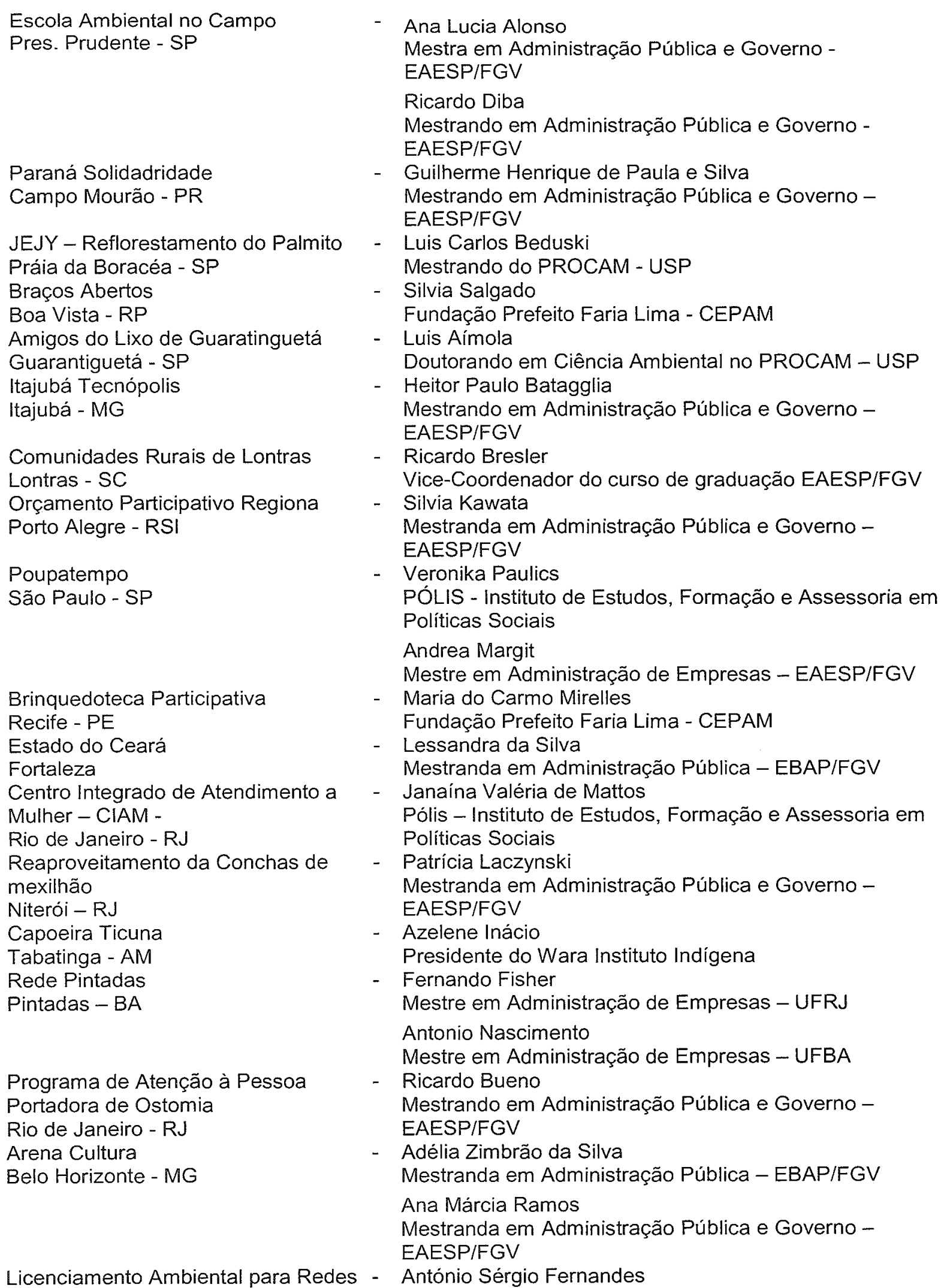


de Infraestrutura

Porto Alegre - RS

Pró - Conecções

Goinésia - GO

Boa Safra

Limoeiro do Norte - CE

Movimento Mulheres

Empreendedoras

Estado do Ceará

Orçamento Participativo Interativo Ipatinga - MG

Fortalecimento da Cadeia Produtiva do Setor Têxtil

Campina Grande - PB

Gestão Democrática da Educação

Cidadania em Cadeia para o Direito do Futuro

Florianópolis - SC

Urbanos

Comissão Perinatal de Belo

Horizonte

Belo Horizonte - MG

Navegar

Rio Amazonas - AM

ANIKE 2002

Camará - PR

Escola Pantaneira

Várias Fazendas - MS
Doutorando em Ciências Políticas - USP

Ferananda Oliveira

Graduanda em Administração Pública - EAESP / FGV

- Mónica Mazzer

Doutoranda em Políticas Sociais - London School of

Economics and Political Science

- Roberta Clemente

Mestre en Administração Pública - EAESP / FGV

- Elaine Gurovitz

Mestrando em Administração Pública e Governo EAESP/FGV

- Antonio José Faria da Costa

Mestrando em Administração Pública e Governo EAESP/FGV

Otávio Prado

Mestrando em Administração Pública e Governo EAESP/FGV

- Leandro Pinheiro

Projeto Saúde e Alegria

- Juliene Azevedo

Mestrando em Administração Pública e Governo EAESP/FGV

- Marco Antonio Carvalho Teixeira

Doutorando em Ciências Sociais - PUC-SP

- Eduardo Caldas

Pólis - Instituto de Estudos, Formação e Assessoria em

Politicas Sociais

Mestre em Administração Pública - EAESP / FGV

Rafael Osório

Graduando em Administração Pública - EAESP / FGV

- Dora Porto

NEPEM - Núcleo de Estudos e Pesquisas sobre a Mulher

- Helio Batista Barbosa

Mestrando em Administração Pública e Governo -

EAESP/FGV

Luis Mario Fujiwara

Mestre em Administração Pública e Governo -

EAESP/FGV

- Fátima Thimoteo

Mestranda em Administração Pública e Governos EAESP/FGV 


\section{FINALISTAS - PROGRAMAS E ÓRGÃOS RESPONSÁVEIS PELAS INSCRIÇÕES}

Amigos do Lixo de Guaratinguetá

ANIKE 2002

Centro Integrado de Atendimento à Mulher

Cidadania em Cadeia para o Direito do

Futuro

Comissão Perinatal de Belo Horizonte

Conselhos Comunitários de Defesa Social do Estado do Ceará

Formação de Parcerias e Geração de Renda nas Comunidades Rurais de Lontras

Licenciamento Ambiental para Redes de Infra-Estrutura

Movimento das Mulheres

Empreendedoras

Orçamento Participativo Interativo

Poupatempo - Central de Atendimento do Cidadão

Pró - Confecções

\section{Boa Safra}

Braços Abertos

Consórcio Intermunicipal de Tratamento de Resíduos Sólidos Urbanos

Programa de Atenção à Pessoa Portadora de Ostomia

Fortalecimento da Cadeia Produtiva do Setor Têxtil

Escola Pantaneira

Jejy - Reflorestamento do Palmito

Rede Pintadas
Secretaria de Serviços Urbanos do Município de Guaratinguetá

Organização dos Professores Indígenas

Conselho Estadual dos Direitos da Mulher

Presídio Masculino de Florianópolis

Secretaria de Saúde do Município de Belo Horizonte

Secretaria de Segurança Pública e Defesa da Cidadania do Estado do Ceará

Secretaria do Desenvolvimento Rural, Agricultura e Meio Ambiente do Município de Lontras

Secretaria de Meio Ambiente do Município de Porto Alegre

Secretaria do Trabalho e Ação Social do Estado do Ceará

Secretaria de Planejamento do Município de Ipatinga

Superintendência Poupatempo

Assessoria de Planejamento da Prefeitura Municipal de Goianésia

Prefeitura Municipal de Limoeiro do Norte

Prefeitura Municipal de Boa Vista

Prefeitura Municipal de Três Passos

Instituto Municipal de Medicina e Reabilitação

Agência Municipal de Desenvolvimento

Secretaria de Planejamento e Desenvolvimento Econômico do Município de Aquidauana

Aldeia Indigena do Ribeirão Silveira

Prefeitura Municipal de Pintadas 